\title{
Systematic Review of Behaviour Change Theories Implementation in Dietary Interventions for People Who Have Survived Cancer
}

\author{
Jana Sremanakova ${ }^{1,2, *(D)}$, Anne Marie Sowerbutts ${ }^{1,2}$, Chris Todd ${ }^{1,2,3,4}$, Richard Cooke ${ }^{5}$ and Sorrel Burden ${ }^{1,2,4,6}$ \\ 1 School of Health Sciences, University of Manchester, Oxford Road, Manchester M13 9PL, UK; \\ annemarie.sowerbutts@manchester.ac.uk (A.M.S.); chris.todd@manchester.ac.uk (C.T.); \\ Sorrel.Burden@manchester.ac.uk (S.B.) \\ 2 Academic Health Science Centre, Manchester M13 9PL, UK \\ Manchester University NHS Foundation Trust, Manchester M13 9WL, UK \\ NIHR Applied Research Collaboration Greater Manchester, Manchester M13 9NQ, UK \\ Department of Psychology, University of Liverpool, Liverpool L69 3B, UK; R.Cooke4@liverpool.ac.uk \\ Salford Royal NHS Foundation Trust, Manchester M6 8HD, UK \\ * Correspondence: jana.sremanakova@manchester.ac.uk; Tel.: +44-(0)-161-306-7673
}

check for

updates

Citation: Sremanakova, J.; Sowerbutts, A.M.; Todd, C.; Cooke, R.; Burden, S. Systematic Review of Behaviour Change Theories Implementation in Dietary Interventions for People Who Have Survived Cancer. Nutrients 2021, 13, 612. https://doi.org/10.3390/ nu13020612

Academic Editors: Hiroto Narimatsu and Yuri Yaguchi

Received: 29 January 2021

Accepted: 10 February 2021

Published: 13 February 2021

Publisher's Note: MDPI stays neutral with regard to jurisdictional claims in published maps and institutional affiliations.

Copyright: (c) 2021 by the authors. Licensee MDPI, Basel, Switzerland. This article is an open access article distributed under the terms and conditions of the Creative Commons Attribution (CC BY) license (https:// creativecommons.org/licenses/by/ $4.0 /)$.

\begin{abstract}
Background: An increasing number of dietary interventions for cancer survivors have been based on the behaviour change theory framework. The purpose of this study is to review the use and implementation of behaviour change theories in dietary interventions for people after cancer and assess their effects on the reported outcomes. Methods: The search strategy from a Cochrane review on dietary interventions for cancer survivors was expanded to incorporate an additional criterion on the use of behaviour change theory and updated to September 2020. Randomised controlled trials (RCT) testing a dietary intervention compared to the control were included. Standard Cochrane methodological procedures were used. Results: Nineteen RCTs, with 6261 participants (age range 44.6 to 73.1 years), were included in the review. The Social Cognitive Theory was the most frequently used theory (15 studies, 79\%). Studies included between 4 to 17 behaviour change techniques. Due to limited information on the mediators of intervention and large heterogeneity between studies, no meta-analyses was conducted to assess which theoretical components of the interventions are effective. Conclusions: Whilst researchers have incorporated behaviour change theories into dietary interventions for cancer survivors, due to inconsistencies in design, evaluation and reporting, the effect of theories on survivors' outcomes remains unclear.
\end{abstract}

Keywords: cancer; survivorship; diet; anthropometry; behaviour change; BCT(s)

\section{Introduction}

The term cancer survivor defines a person living with and beyond cancer [1]. In this article, we define cancer survivors as people who completed all the active treatments and are in the recovery period. Adherence to a healthy lifestyle, including eating a healthy diet and being physically active, has been associated with a reduction in overall mortality among cancer survivors [2,3]. It has been suggested that the experience of cancer diagnosis and cancer treatment stimulates survivors' motivation to change their lifestyle [4]. This is supported by qualitative studies indicating that people are willing to make changes to their lifestyle and have different needs and preferences for support after treatment [5-9]. Hence, identification of the most effective approaches to help people who experienced cancer change their lifestyle is crucial for achieving improvement in their health outcomes.

There is a growing interest in utilising behaviour change theories to inform the content and design of interventions. A large body of scientific evidence suggests that interventions addressing change in health behaviours are more likely to achieve success if they are 
developed with a clear understanding of the targeted behaviour, its environmental context, and if the intervention incorporates a theoretical basis [10-14].

A number of systematic reviews have identified the most frequently used theories in health interventions [15-17]. These reviews show that the selection of theories has not changed over the last 20 years, with the most frequently used theories being Social Cognitive Theory (SCT) [18], the Trans Theoretical Model (TTM; also known as the Stages of Change) [19], and the Health Belief Model (HBM) [20].

The extent to which theories are implemented within interventions varies. An intervention can be classified as either informed by theory, applying theory, testing theory, building or creating theory [14]. In addition, theories can be applied at different levels targeting individuals, groups, organisations or communities [21].

A number of concerns have been raised around the validity and reliability of theories used to address health behaviour, their application, interpretation, and translation into applied research and clinical practice. In summary, a number of researchers [22-26] have drawn attention to "how we use theory, how we test theory, how we translate theories into interventions, and what conclusions we draw from research" [14] and ask for clarification and transparency of behaviour change interventions [24].

To understand how theoretical components work in an intervention, there is a need for authors to provide a detailed report of how the theory has been used to inform the intervention. Moreover, there should be an identification of the components of the intervention using a taxonomy of behaviour change techniques (BCTs) $[11,22,27]$, such as the one reported by Michie et al. (2013) [28]. This can help with the understanding of behaviour change tools used in the interventions. Although, BCTs are not directly linked with a specific theory, they can have implications for theory when there is an obvious link between the theory and BCTs.

When theory is applied to interventions, it is important to conduct both process evaluation, alongside the usual outcome evaluation. The outcome evaluation tests the efficacy of an intervention, by looking for positive changes in measures of physiological (body fat), health (survival), and behavioural outcomes (fruit intake). Although sometimes studies depend on surrogate outcomes, such as the intention to change behaviour, which are mediators or determinants of behaviour. The process evaluation identifies how the intervention is designed to work and how changes in the determinants of behaviour (e.g., self-efficacy) [24] mediate the effect of the intervention on outcomes.

In cancer survivorship research, encouraging results have been demonstrated in systematic reviews of interventions that have utilised the behaviour change theory (ies) to increase exercise [22] and improve the quality of life [29]. In dietary interventions for cancer survivors, only one study assessed using dietary changes as outcomes for SCT based interventions and indicated promising improvement in dietary behaviour [27]. Since then, theories have been widely used to inform the design and development of behaviour change interventions [30]. To date, however, little is known about the degree of use and implementation of behaviour change theories and BCTs in dietary interventions for cancer survivors. Hence, the aims of this review are as follows: (1) To identify what theories have been used; (2) to establish the extent of implementation of behaviour change theories and BCTs; (3) to identify what behaviour change outcomes are reported; and (4) to report on the effects of theories and BCTs on outcomes in dietary interventions for people after cancer.

\section{Materials and Methods}

This systematic review was registered with PROSPERO, number CRD42020172444 and follows the standard of Cochrane systematic reviews [31] and the Preferred Reporting Items for Systematic Reviews and Meta-analysis (PRISMA) [32].

\subsection{Literature Searching}

This systematic review extends the information collected for the Cochrane review on dietary interventions for cancer survivors [30]. During the completion of the Cochrane 
review, the question on implementation of behaviour change theory emerged. However, behaviour change was not within the scope of the review and agreed protocol. Searches conducted for a previous Cochrane review were updated on 30 September 2020 and an additional inclusion criterion on the use of behaviour change theory was incorporated. We have included only RCTs, however, 11 trials are final reports of large projects [33-43] that included non-randomised studies and qualitative work. The following databases were searched: The Cochrane Central Register of Controlled trials; Medline via Ovid; Embase via Ovid; the Allied and Complementary Medicine Database; the Cumulative Index to Nursing and Allied Health Literature; and the Database of Abstracts of Reviews of Effects. We also searched other resources, other reviews on the topic, and the International Trials Registry for ongoing trials. Search words were matched with the MESH term truncation systems and Boolean operators "and" with "or" function were used. The search strategy is attached as Supplementary Table S1. The results of the literature searches were uploaded to Covidence, (https: / / www.covidence.org/ (accessed on 10 December 2020), Melbourne, AU) an online software for data management. Data were checked for duplicates by the software and then manually. The titles and abstracts were independently screened by both Jana Sremanakova and Sorrel Burden based on inclusion and exclusion criteria. Full texts were obtained to identify the eligible publications and checked by both Jana Sremanakova and Anne Marie Sowerbutts Any discrepancy during the process of screening was discussed between Jana Sremanakova, Sorrel Burden, and Anne Marie Sowerbutts. The search results and selection process have been recorded in the PRISMA (Preferred Reporting Items for Systematic Reviews and Meta-Analyses) flow diagram (Figure 1).

\subsection{Study Inclusion Criteria}

The primary criterion for this review was the use of behaviour change theory in the intervention design. Participants were adult (age $>18$ ) survivors of cancer who completed surgery and all anticancer treatments. Only randomised control trials (RCT) testing a dietary intervention compared to a control group following standard care were included. The dietary intervention was defined as an oral nutritional intervention based only on promoting a healthy diet. Interventions were excluded if they were based on a single food group, oral supplements, including those with single or multiple nutrients and probiotic supplements, as well as studies using intravenous nutrient solutions including both enteral and parenteral nutrition. Dietary interventions using any delivery method: Group sessions, telephone instruction, written materials, mobile application, or web-based approaches were included.

\subsection{Outcomes}

The main outcomes of interest were the dietary behaviours and surrogate outcomes. Dietary behaviour included changes in energy consumption, nutrients, food groups identified by using food frequency questionnaires, dietary recall, food diaries or assessed by dietary assessment methods, including changes in anthropometry and body composition. Mediators of behaviour (surrogate outcomes) included psychological constructs (questionnaire-based score assessing constructs such as self-efficacy, intention or similar), readiness to change and goal settings (number of goals set).

\subsection{Data Extraction and Risk of Bias Assessment}

A data extraction form was devised based on a Cochrane template [30]. One author (Jana Sremanakova) extracted data on study characteristics (author, publication year, full title, location, funding, study design, and duration), population characteristics (age, gender, type of treatment, cancer site, and stage), cancer site, dietary intervention provided, behaviour change theory, level of implementation of behaviour change approach in design, intervention resources, targeted behaviour or constructs, measured behaviour or constructs, form of testing behaviour change approach, outcomes of behavioural change, and adherence measures. Twenty percent of data extraction was double checked by all 
the co-authors. The BCT taxonomy [28] was used to identify the BCTs in the interventions. Jana Sremanakova independently coded BCTs for all the publications. In addition, all the co-authors coded $25 \%$ of publications and all the authors discussed with JS a final selection of BCTs. The risk of bias was assessed using the Cochrane Collaboration's risk of bias tool [44]. The quality of evidence was generally low to very low, however, some outcomes were assessed as being of a moderate-certainty of evidence using GRADE [45] (See Supplementary Tables S2 and S3 for the GRADE assessment, and Figure 2 for the assessed risk of bias).

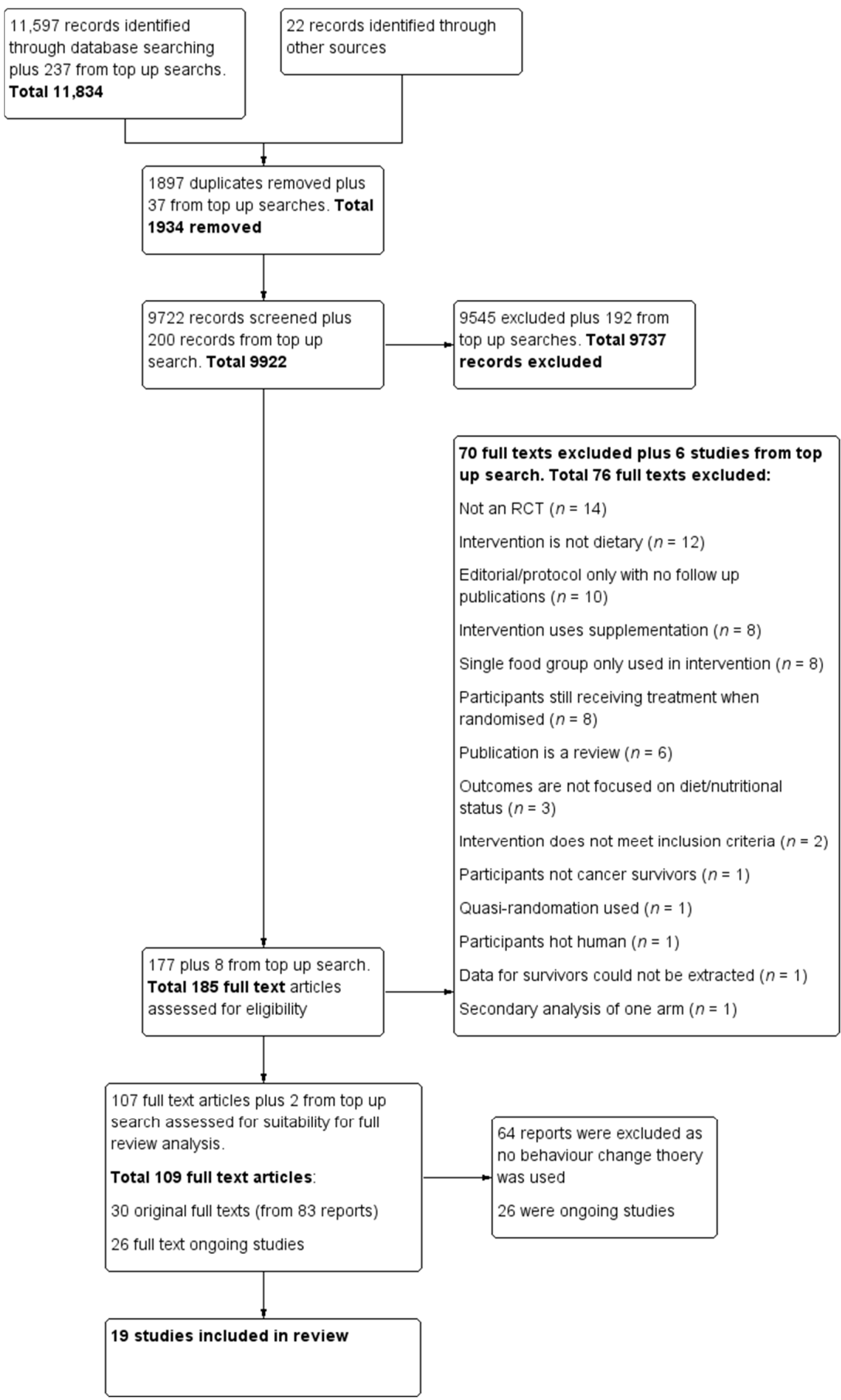

Figure 1. Preferred reporting items for systematic reviews and meta-analysis (PRISMA) flow diagram for the study selection. 


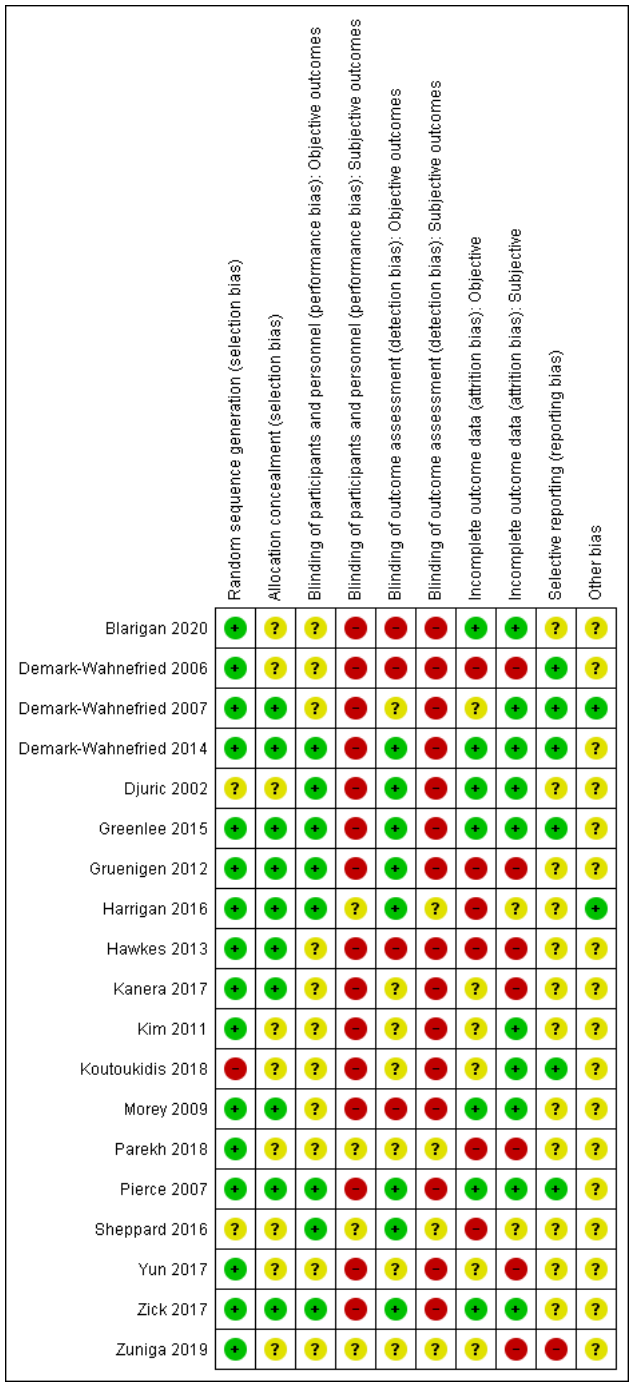

Figure 2. Risk of bias summary for each included study. Note: green (+)—low risk of bias, red (-)-high risk of bias, yellow (?)-unclear risk of bias.

\subsection{Statistical Methods}

We used standard Cochrane methodological procedures and completed the metaanalysis in the Cochrane Review manger version 5.4.1 software (UK) [46]. The mean difference (MD) and 95\% confidence intervals (CI) were calculated using a random effect statistical model. Heterogeneity of any combined studies was assessed by using $\mathrm{I}^{2}$ (heterogeneity). If $\mathrm{I}^{2}$ was greater than $30 \%$, we examined possible reasons for heterogeneity in relation to study participants and similarity of clinical parameters in studies. Data not suitable for the meta-analysis were reported narratively.

\section{Results}

\subsection{Search Results}

Nineteen studies met the inclusion criteria and were included in this review with two studies identified based on the updated search.

\subsection{Studies Characteristics}

A total of 6261 participants were included in studies on dietary interventions for cancer survivors which utilised behaviour change theory (ies). The mean age of participants reported was between 44.6 to 73.1 years old. The majority of studies recruited women with breast cancer, so the proportion of male $(982,16 \%)$ and female $(5279,84 \%)$ participants 
was unequal. Ten studies (53\%) included participants after breast cancer and two studies combined breast and prostate cancer. Two studies focused on gynaecological cancer, two on colon cancer, and three studies included a mixed population. Fourteen studies were conducted in the USA, two studies in South Korea, one study in Australia, one in the United Kingdom, and one in the Netherlands. Details on participant characteristics are reported in Table 1.

Table 1. Participant characteristics.

\begin{tabular}{|c|c|c|c|c|c|c|}
\hline Authors & $n$ & $\begin{array}{l}\text { Mean Age } \\
\text { (SD) (Years) }\end{array}$ & $\begin{array}{l}\text { Gender } \\
\text { (F:M ratio) }\end{array}$ & $\begin{array}{l}\text { Ethnicity } \\
(\%)\end{array}$ & $\begin{array}{l}\text { Higher education } \\
(\%)\end{array}$ & $\begin{array}{l}\text { Cancer } \\
\text { Site }\end{array}$ \\
\hline Blarigan 2020 [47] & 50 & $5.5(3.5)$ & $33 / 17$ & White $70 \%$, Other $30 \%$ & College graduate $96 \%$ & Colorectal \\
\hline $\begin{array}{l}\text { Demark- } \\
\text { Wahnefried } \\
2006 \text { [34] }\end{array}$ & 182 & $\begin{array}{l}71.5(4.4) \mathrm{vs} . \\
71.9(5.6)\end{array}$ & $104 / 78$ & $\begin{array}{l}\text { White } 82.0 \% \text { vs. } 82.8 \% \\
\text { African American } 14.6 \% \text { vs. } \\
15.0 \% \text {, Other } 3.4 \% \text { vs. } 2.2 \%\end{array}$ & Not reported & Breast, prostate \\
\hline $\begin{array}{l}\text { Demark- } \\
\text { Wahnefried } \\
2007[33]\end{array}$ & 543 & $57(10.8)$ & $304 / 239$ & White $83 \%$, Black $13 \%$, Other $4 \%$ & $\begin{array}{l}\text { College graduate/ } \\
\text { post graduate } 58 \%\end{array}$ & Breast, prostate \\
\hline $\begin{array}{c}\text { Demark- } \\
\text { Wahnefried } \\
2014 \text { [35] }\end{array}$ & 43 & $61.3(7.4)^{* *}$ & $43 / 0$ & $\begin{array}{c}\text { Non-Hispanic White } 74 \% \\
\text { Hispanic white } 7 \% \text {, African } \\
\text { American } 18 \% \text {, Asian } 1 \%\end{array}$ & College graduate $34.3 \%$ & Breast \\
\hline $\begin{array}{l}\text { Djuric } \\
2002[36]\end{array}$ & 48 & $36-70 *$ & $48 / 0$ & $\begin{array}{c}\text { White } 73 \% \text {, African } \\
\text { American } 25 \%\end{array}$ & College graduate $63 \%$ & Breast \\
\hline $\begin{array}{l}\text { Greenlee } \\
2015[37]\end{array}$ & 70 & $\begin{array}{l}55.1(9.1) \text { vs. } \\
58.0(10.1)\end{array}$ & $70 / 0$ & $\begin{array}{c}\text { White } 41.2 \% \text { vs. } 38.9 \% \text {, Black } 20.6 \% \\
\text { vs. } 30.6 \% \text {, Mixed } 14.7 \% \text { vs. } 16.7 \% \text {, } \\
\text { Native American } 5.9 \% \text { vs. } 0.0 \%\end{array}$ & $\begin{array}{c}\text { College degree or higher } 14.7 \% \\
\text { vs. } 5.6 \%\end{array}$ & Breast \\
\hline $\begin{array}{l}\text { Gruenigen } \\
2012[43]\end{array}$ & 75 & $\begin{array}{l}57(8.6) \text { vs. } \\
58.9(10.9)\end{array}$ & $75 / 0$ & $\begin{array}{c}\text { Caucasian } 87.8 \% \text { vs. } 94.1 \% \\
\text { African American } 9.8 \% \text { vs. } 2.9 \% \text {, } \\
\text { Other } 2.4 \% \text { vs. } 2.9 \%\end{array}$ & $\begin{array}{l}\text { College graduate or higher } \\
39.0 \% \text { vs. } 41.2 \%\end{array}$ & $\begin{array}{l}\text { Gynaeco- } \\
\text { logical }\end{array}$ \\
\hline $\begin{array}{l}\text { Harrigan } \\
2016[38]\end{array}$ & 100 & $59(7.5)$ & $100 / 0$ & Non-Hispanic White $91 \%$ & $\begin{array}{c}\text { College degree } 29 \% \text {, graduate } \\
\text { degree } 37 \%\end{array}$ & Breast \\
\hline $\begin{array}{l}\text { Hawkes } \\
2013\end{array}$ & 410 & $\begin{array}{l}64.9(10.8) \text { vs. } \\
67.8(9.2)\end{array}$ & $189 / 221$ & Not reported & Not reported & Colon \\
\hline $\begin{array}{l}\text { Kanera } \\
2017[48]\end{array}$ & 462 & $\begin{array}{l}55.6(11.5) \text { vs. } \\
56.2(11.3)\end{array}$ & $369 / 93$ & Not reported & High education $34.2 \%$ vs. $27.7 \%$ & Mix \\
\hline$\underset{2011[49]}{\operatorname{Kim}}$ & 45 & $\begin{array}{l}44.6(9.9) \text { vs. } \\
47.1(7.3)\end{array}$ & $45 / 0$ & Not reported & Completed university $35.5 \%$ & Breast \\
\hline Koutoukidis 2019 [40] & 62 & $62.1(8.3)$ & $62 / 0$ & $\begin{array}{c}\text { White } 67 \% \text { Asian } 18 \% \text { Black } 8 \% \\
\text { Other } 6 \%\end{array}$ & Degree $47 \%$ & $\begin{array}{l}\text { Gynaeco- } \\
\text { logical }\end{array}$ \\
\hline $\begin{array}{l}\text { Morey } \\
2009[41]\end{array}$ & 641 & $73(5.0)$ vs. $73.1(5.1)$ & $349 / 292$ & White $89.0 \%$ vs. $88.5 \%$ & Not reported & $\operatorname{Mix}^{\circ}$ \\
\hline $\begin{array}{c}\text { Pierce } \\
2007[42]\end{array}$ & 3088 & $\begin{array}{l}53.3(9.8) \text { vs. } \\
53.0(9.0)\end{array}$ & $3088 / 0$ & $\begin{array}{c}\text { Asian } 3.6 \% \text { vs. } 6.5 \% \text {, Black or } \\
\text { African American } 28.6 \% \text { vs. } 16.1 \% \text {, } \\
\text { White } 67.9 \% \text { vs. } 67.7, \text { American } \\
\text { Indian / Alaska Native } 0.0 \% \text { vs. } \\
6.5 \% \text {, Other race } 0 \text { vs. } 3.2 \% \\
\text { White } 85 \% \text { vs. } 85.6 \% \text {, African } \\
\text { American } 4 \% \text { vs. } 3.7 \% \text {, Hispanic } \\
5.7 \% \text { vs. } 5 \% \text {, Asian American } 3 \% \\
\text { vs. } 3.2 \% \text {, Mixed /other } 2.3 \% \\
\text { vs. } 2.5 \%\end{array}$ & $\begin{array}{l}\text { BA } 25 \% \text { vs. } 35.5 \% \text {, MA } 39.3 \% \\
\text { vs. } 35.5 \%, \operatorname{PhD} 3.6 \%\end{array}$ & Breast \\
\hline $\begin{array}{l}\text { Sheppard } \\
2016[51]\end{array}$ & 22 & $54.7(9.8)$ & $22 / 0$ & African American 100\% & Not reported & Breast \\
\hline $\begin{array}{l}\text { Yun } \\
2017[52]\end{array}$ & 206 & $50.68(9.4)$ & $164 / 42$ & Korean $100 \%$ & College $48.53 \%$ & $\operatorname{Mix}^{\circ}$ \\
\hline $\begin{array}{c}\text { Zick } \\
2017[53]\end{array}$ & 30 & $\begin{array}{l}64.4(10.0) \text { vs. } \\
10.4(9.35)\end{array}$ & $30 / 0$ & White $93 \%$ & Not reported & Breast \\
\hline $\begin{array}{l}\text { Zuniga } \\
2019[50]\end{array}$ & 125 & $\begin{array}{l}55.3(10.3) \text { vs. } \\
58.4(8.2)\end{array}$ & $125 / 0$ & $\begin{array}{c}\text { Anglo } 41.7 \% \text { vs. } 43.1 \% \\
\text { Latino } 51.7 \% \text { vs. } 50.8 \% \\
\text { Other } 6.7 \% \text { vs. } 6.2 \%\end{array}$ & $\begin{array}{c}\text { Some college/degree } 40.0 \% \text { vs. } \\
26.2 \% \text { college or higher } 45.0 \% \\
\text { vs. } 63.1 \%\end{array}$ & Breast \\
\hline
\end{tabular}

Note: Intervention versus control was reported where the overall mean is not present; ${ }^{*}$ range only report; ${ }^{\circ}$ breast, colon, prostate, and other cancer sites; ${ }^{* *}$ calculation includes individual and group arm; $n$-total number of participants.

Most studies used a combination of strategies including mailed intervention, telephone calls, group sessions, individual sessions, automated messages, web-based interventions, and newsletters. However, there was always a predominant strategy: Eight studies used group interventions [36,37,40,43,50-52,54], four studies used mailed interventions [33-35,41], three studies used telephone counselling [39,42,49], two studies used individual sessions [38,53], and two studies were a web-based intervention [47,48]. One study [33] considered the ethnic difference in the study population by tailoring the resources based on age, race, and style of coping with cancer such as cognitive avoider, helpless or hopeless. There was one study that focused on black ethnic survivors of breast cancer [51], one study on the 
Korean population [52], and one study developed the evidence-based programme targeting Hispanic breast cancer survivors [37].

Although the included studies were all dietary interventions, most ( $74 \%, 14$ studies) also targeted changes in physical activity. The length of interventions ranged from 1.8 to 12 months of follow up, but one study had 7.3 years of follow up [42]. The dropout rate was $11 \%$ (692 out of 6407 participants randomised).

Seven studies used an attention control group and provided participants with untailored information booklets [33-35,37,53], newsletters, and cooking classes [42] or suggested participants to follow a weight loss programme on their own [36]. Six studies used a waiting list control group and provided participants with general information [41,47,49-52]. Six studies used the usual care group with no additional support [38-40,43,48,54].

Only eight studies $(42 \%)$ published a protocol with a detailed description of the intervention [33,34,37,39-42,54]. Four studies (21\%) did not publish a protocol but included a description of the intervention in the main paper [36,48,49,51]. Additionally, seven studies $(37 \%)$ provided limited information on the intervention $[35,38,43,47,50,52,53]$.

\subsection{Theoretical Framework}

\subsubsection{Implementation of Behaviour Change Theory in the Interventions}

All 19 studies specified at least one theoretical framework. Social Cognitive Theory (SCT) was the most frequently used theory (15 studies, 79\%), followed by the TransTheoretical Model of Change (TTM; nine studies, 47\%), then the Theory of Planned Behaviour (TPB, three studies, $16 \%$ ). One study reported using the Acceptance Commitment Model (ACM) and another reported using the Control Theory (CT). Ten studies (53\%) based their intervention on two theories, most frequently SCT and the TTM. Eight studies $(42 \%)$ used one theory, while one study used three theories (SCT, TPB, TTM). In addition to these theories, the Motivational Interviewing Technique (MIT) was used in three studies (three studies, $16 \%$ ) $[39,51,54]$. The studies targeted changes in one or several constructs as part of the intervention. The most frequently targeted constructs were self-efficacy to gain confidence in the participants' ability to change their lifestyle, goal settings targeting ability to select achievable goals, and behavioral capacity focusing on knowledge of a healthy lifestyle. Details of the studies' characteristics are included in Table 2.

Table 2. Characteristics of the intervention.

\begin{tabular}{|c|c|c|c|c|c|c|c|}
\hline Authors & Theory & $\begin{array}{l}\text { Intervention Design } \\
\text { and Resources }\end{array}$ & $\begin{array}{c}\text { Behavioural } \\
\text { Mediators } \\
\text { Measured }\end{array}$ & $\begin{array}{c}\text { Dietary Behaviour } \\
\text { Beasures }\end{array}$ & $\begin{array}{l}\text { Dietary } \\
\text { Assessment } \\
\text { Method }\end{array}$ & $\begin{array}{l}\text { Physical } \\
\text { Activity }\end{array}$ & $\begin{array}{l}\text { Time Point } \\
\text { (Months) }\end{array}$ \\
\hline $\begin{array}{l}\text { Blarigan } \\
2020[47]\end{array}$ & SCT, TPB & $\begin{array}{l}\text { Web based-text messages, printed } \\
\text { materials, and personalised reports }\end{array}$ & Not reported & $\begin{array}{l}\text { Vegetables, grains, } \\
\text { fish, meat, sugary } \\
\text { drinks, alcohol }\end{array}$ & $\begin{array}{l}24 \mathrm{~h} \text { recall for } \\
4 \text { days }\end{array}$ & No & 3,6 follow up \\
\hline $\begin{array}{c}\text { Demark- } \\
\text { Wahnefried } \\
2006 \text { [34] }\end{array}$ & TTM, SCT & $\begin{array}{l}\text { Paper based-mailed information, } \\
\text { telephone counselling, tailored } \\
\text { workbook-information on current } \\
\text { stage of diet/exercise, comparison } \\
\text { to national guide and tips } \\
\text { for change }\end{array}$ & $\begin{array}{l}\text { Readiness to } \\
\text { healthy diet, } \\
\text { self-efficacy to } \\
\text { healthy diet }\end{array}$ & $\begin{array}{l}\text { Fruit and } \\
\text { vegetables, } \\
\text { BMI, DQI }\end{array}$ & 3-day recall & Yes & 6,12 follow up \\
\hline $\begin{array}{c}\text { Demark- } \\
\text { Wahnefried } \\
2007 \text { [33] }\end{array}$ & TTM, SCT & $\begin{array}{l}\text { Paper based-mailed information, } \\
\text { customized messages based on IT } \\
\text { system programme reflecting TTM, } \\
\text { SCT in tailored workbook, record } \\
\text { logs, newsletter, advice for } \\
\text { overcoming barriers, fun facts, } \\
\text { graphic depiction of progress, } \\
\text { update cards, pedometers }\end{array}$ & $\begin{array}{l}\text { Self-efficacy of } \\
\text { eating fruits and } \\
\text { vegetables, stage } \\
\text { of readiness to } \\
\text { increase fruits } \\
\text { and vegetables, } \\
\text { behaviours } \\
\text { practised at } \\
\text { goal level }\end{array}$ & $\begin{array}{c}\text { Fruit and } \\
\text { vegetables, BMI, } \\
\text { Fat (kcal), DQI }\end{array}$ & $\begin{array}{l}\text { Diet history } \\
\text { questionnaire }\end{array}$ & Yes & 10 \\
\hline $\begin{array}{c}\text { Demark- } \\
\text { Wahnefried } \\
2014 \text { [35] }\end{array}$ & SCT, TTM & $\begin{array}{l}\text { Mailed intervention-SCT tailored } \\
\text { newsletters, messages on progress, } \\
\text { reinforcement, encouragement, } \\
\text { feedback, barriers, shoe chip, food } \\
\text { records, activities logs, logbook, } \\
\text { reference manual, website }\end{array}$ & $\begin{array}{l}\text { Self-efficacy of } \\
\text { adhering to } \\
\text { healthy weight } \\
\text { loss diet }\end{array}$ & $\begin{array}{l}\text { EI, BMI, weight, } \\
\text { WC, DQI }\end{array}$ & $24 \mathrm{~h}$ recall & Yes & 12 \\
\hline
\end{tabular}


Table 2. Cont.

\begin{tabular}{|c|c|c|c|c|c|c|c|}
\hline Authors & Theory & $\begin{array}{l}\text { Intervention Design } \\
\text { and Resources }\end{array}$ & $\begin{array}{l}\text { Behavioural } \\
\text { Mediators } \\
\text { Measured }\end{array}$ & $\begin{array}{c}\text { Dietary Behaviour } \\
\text { Beasures }\end{array}$ & $\begin{array}{l}\text { Dietary } \\
\text { Assessment } \\
\text { Method }\end{array}$ & $\begin{array}{l}\text { Physical } \\
\text { Activity }\end{array}$ & $\begin{array}{l}\text { Time Point } \\
\text { (Months) }\end{array}$ \\
\hline $\begin{array}{c}\text { Djuric } \\
2002[36]\end{array}$ & SCT & $\begin{array}{l}\text { One to one/telephone counselling, } \\
\text { group meetings, written resources } \\
\text { but details not reported }\end{array}$ & Not reported & EI & $\begin{array}{l}\text { 3-day } \\
\text { food diary }\end{array}$ & Yes & 3,12 \\
\hline $\begin{array}{l}\text { Greenlee } \\
2015[37]\end{array}$ & TTM, SCT & $\begin{array}{l}\text { Group sessions on education, } \\
\text { cooking and healthy shopping, } \\
\text { resources-no details }\end{array}$ & Not reported & $\begin{array}{l}\text { EI, fruit and } \\
\text { vegetables, weight, } \\
\text { BMI, WC, } \\
\text { HC, WHP }\end{array}$ & $24 \mathrm{~h}$ recall & No & 3,6 follow up \\
\hline $\begin{array}{l}\text { Gruenigen } \\
2012 \text { [43] }\end{array}$ & SCT & $\begin{array}{l}\text { Group sessions, follow up } \\
\text { newsletter, telephone, and emails to } \\
\text { reinforce goals, } \\
\text { resources-no detials }\end{array}$ & Not reported & $\begin{array}{l}\text { fruit and } \\
\text { vegetables, EI, } \\
\text { weight, WC }\end{array}$ & $24 \mathrm{~h}$ recall & Yes & 6,12 follow up \\
\hline $\begin{array}{l}\text { Harrigan } \\
2016[38]\end{array}$ & SCT & $\begin{array}{l}\text { One to one counselling, pedometer, } \\
\text { scale, LEAN book-no details }\end{array}$ & Not reported & $\begin{array}{l}\text { fruit and } \\
\text { vegetables, weight, } \\
\text { WC, \% fat }\end{array}$ & FFQ & Yes & 6,12 follow up \\
\hline $\begin{array}{l}\text { Hawkes } \\
2013[39]\end{array}$ & $\begin{array}{l}\text { ACM, } \\
\text { MIT }\end{array}$ & $\begin{array}{l}\text { Telephone delivered health } \\
\text { coaching sessions; postcard } \\
\text { prompts; pedometer, book with } \\
\text { educational information on } \\
\text { lifestyle behaviours }\end{array}$ & Not reported & $\begin{array}{l}\text { Fibre, fruit and } \\
\text { vegetables, } \\
\text { alcohol, BMI }\end{array}$ & FFQ & Yes & 6,12 follow up \\
\hline $\begin{array}{l}\text { Kanera } \\
2017[48]\end{array}$ & $\begin{array}{l}\text { SCT, TPB, } \\
\text { SRT, IMC }\end{array}$ & $\begin{array}{l}\text { Web-based programme with } \\
\text { personalised feedback, } \\
\text { online-evaluation session at the end }\end{array}$ & Not reported & Vegetable intake & $\begin{array}{l}\text { Dutch } \\
\text { standard } \\
\text { questionnaire }\end{array}$ & Yes & 12 follow up \\
\hline$\underset{2011[49]}{\operatorname{Kim}}$ & TTM & $\begin{array}{c}\text { Telephone counselling, workbook } \\
\text { on diet and exercise, heart } \\
\text { rate monitor }\end{array}$ & $\begin{array}{l}\text { Readiness to } \\
\text { change }\end{array}$ & DQI & $\begin{array}{l}\text { 3-day } \\
\text { recall }\end{array}$ & Yes & 3 \\
\hline $\begin{array}{l}\text { Koutoukidis } \\
2018 \text { [40] }\end{array}$ & SCT, CT & $\begin{array}{l}\text { Group based intervention on eating } \\
\text { pattern, balanced diet, portion size, } \\
\text { food triggers, food labels and } \\
\text { physical activity, study manual }\end{array}$ & Not reported & $\begin{array}{l}\text { Healthy Eating } \\
\text { Index }\end{array}$ & $\begin{array}{l}24 \mathrm{~h} \\
\text { recall }\end{array}$ & Yes & $\begin{array}{l}1.8,5.5 \\
\text { follow up }\end{array}$ \\
\hline $\begin{array}{c}\text { Morey } \\
2009[41]\end{array}$ & $\begin{array}{l}\text { TTM, } \\
\text { SCT }\end{array}$ & $\begin{array}{l}\text { Paper based tailored workbook, } \\
\text { newsletters, SCT telephone } \\
\text { counselling, automated prompts, } \\
\text { pedometer, exercise bands, table } \\
\text { guide, record logs, workbook on } \\
\text { diet and exercise }\end{array}$ & Not reported & $\begin{array}{c}\text { Fruit and } \\
\text { vegetables, } \\
\text { weight, BMI }\end{array}$ & $\begin{array}{l}24 \mathrm{~h} \\
\text { recall }\end{array}$ & Yes & 12 \\
\hline $\begin{array}{l}\text { Parekh } \\
2018[50]\end{array}$ & SCT & $\begin{array}{l}\text { Group education sessions on diet, } \\
\text { exercise and cooking classes, } \\
\text { information brochures-no details }\end{array}$ & Not reported & $\begin{array}{l}\text { Fruit and } \\
\text { vegetables }\end{array}$ & $\begin{array}{l}\text { Validated tool } \\
\text { for fruit and } \\
\text { vegetables }\end{array}$ & Yes & 3 \\
\hline $\begin{array}{c}\text { Pierce } \\
2007[42]\end{array}$ & SCT & $\begin{array}{l}\text { Telephone counselling sessions, } \\
\text { cooking classes, } \\
\text { newsletters-no details }\end{array}$ & Not reported & $\begin{array}{l}\text { EI, fruit and } \\
\text { vegetables, fibre, } \\
\text { weight, adherence }\end{array}$ & $\begin{array}{l}24 \mathrm{~h} \\
\text { recall }\end{array}$ & No & 72 \\
\hline $\begin{array}{l}\text { Sheppard } \\
2016[51]\end{array}$ & $\begin{array}{c}\text { SCT, } \\
\text { TPB, MIT }\end{array}$ & $\begin{array}{l}\text { Group session-SCT, TBP, MIT } \\
\text { telephone sessions, pedometers, } \\
\text { notebook, resources-no details }\end{array}$ & Not reported & $\begin{array}{l}\text { EI, fibre, weight, } \\
\text { BMI, WC, } \\
\text { HC, WHR }\end{array}$ & $\begin{array}{l}\text { 4-day food } \\
\text { diary }\end{array}$ & Yes & 3,12 \\
\hline $\begin{array}{l}\text { Yun } \\
2017[52]\end{array}$ & TTM & $\begin{array}{l}\text { Educational workshop, individual } \\
\text { telephone coaching, partnership } \\
\text { with cancer survivors, } \\
\text { resources-no details }\end{array}$ & Not reported & Vegetable intake & $\begin{array}{c}\text { Validated } \\
\text { questionnaire }\end{array}$ & Yes & 12 \\
\hline $\begin{array}{c}\text { Zick } \\
2017[53]\end{array}$ & SCT & $\begin{array}{c}\text { Individualised telephone } \\
\text { counselling, self-monitoring } \\
\text { check list }\end{array}$ & Not reported & $\begin{array}{l}\text { EI, vegetable } \\
\text { intake, BMI }\end{array}$ & $24 \mathrm{~h}$ recall & No & 3 \\
\hline $\begin{array}{l}\text { Zuniga } \\
2019[54]\end{array}$ & TTM, MIT & $\begin{array}{l}\text { Workshops with cooking } \\
\text { demonstration, MIT telephone calls, } \\
\text { newsletter, copies of lectures, TTM } \\
\text { based sheet with goals }\end{array}$ & Not reported & $\begin{array}{l}\text { EI, fibre, fruit and } \\
\text { vegetables }\end{array}$ & $\begin{array}{l}\text { Mediterranean } \\
\text { diet } \\
\text { questionnaire }\end{array}$ & No & 6 \\
\hline
\end{tabular}

Note: ACM-Acceptance Commitment Model, BMI—body mass index, DQI-Diet quality index, EI-energy intake, FFQ_food frequency questionnaire, HC - hip circumference, IMC — Integrated Model of Change, MIT_Motivational Interviewing, SCT—Social Cognitive Theory, SRT-Self-regulation theory, TTM-Trans-Theoretical Model of Change, TBP-Theory of Planned Behaviour, WC-waist circumference, WHR - waist o hip ratio.

\subsubsection{Use and Reporting of Behaviour Change Techniques in the Interventions}

The quality of reported details on how the intervention was performed varied between studies, and influenced our ability to map the BCTs used in the interventions. Based on the BCT taxonomy classification, interventions focused on the following BCTs: 9.1. Credible source (19 studies), 4.1. Instruction on how to perform the behaviour (18 studies), 3.1. Social support (17 studies), 1.3. Goal setting (12 studies), 2.3. Self-monitoring of behaviour (12 studies), 1.2. Problem solving (10 studies), and 2.2. Feedback on behaviour (10 studies). The studies used between four to 17 BCTs (see Table 3). 
Table 3. Behaviour change techniques taxonomy mapping of the studies.

\begin{tabular}{|c|c|c|c|c|c|c|c|c|c|c|c|c|c|c|c|c|c|c|c|c|}
\hline $\begin{array}{l}\text { вст } \\
\text { no. }\end{array}$ & BCTs/Authors & $\begin{array}{l}\text { Blarigan } \\
2020 \text { (47] }\end{array}$ & $\begin{array}{c}\text { Demark- } \\
\text { Wahnefried } \\
2006[34]\end{array}$ & $\begin{array}{c}\text { Demark- } \\
\text { Wahnefrried } \\
2007[33]\end{array}$ & $\begin{array}{c}\text { Demark- } \\
\text { Wahnefried } \\
2014[35]\end{array}$ & $\begin{array}{c}\text { Djuric } \\
2002[36]\end{array}$ & $\begin{array}{l}\text { Greenlee } \\
2015[37]\end{array}$ & $\begin{array}{l}\text { Gruenigen } \\
2012[43]\end{array}$ & $\begin{array}{l}\text { Harrigan } \\
2016[31]\end{array}$ & $\begin{array}{l}\text { Hawkes } \\
2013[39]\end{array}$ & $\begin{array}{c}\text { Kanera } \\
2017 \text { [48] }\end{array}$ & $\underset{2011[49]}{\operatorname{Kim}}$ & $\begin{array}{l}\text { Koutoukidis } \\
2019 \text { [40] }\end{array}$ & $\begin{array}{l}\text { Morey } \\
2009 \text { [41] }\end{array}$ & $\begin{array}{c}\text { Parekh } \\
2018[50]\end{array}$ & $\begin{array}{c}\text { Pierce } \\
2007 \text { [42] }\end{array}$ & $\begin{array}{l}\text { Sheppard } \\
2016[51]\end{array}$ & $\begin{array}{c}\text { Yun } \\
2017 \text { [52] }\end{array}$ & $\begin{array}{c}\text { Zick } \\
2017 \text { [53] }\end{array}$ & $\begin{array}{c}\text { Zuniga } \\
2019 \text { [54] }\end{array}$ \\
\hline $\begin{array}{l}1.1 \\
1.2\end{array}$ & $\begin{array}{l}\text { Goal setting (behaviour) } \\
\text { Problem solving }\end{array}$ & 1 & 1 & ${ }_{1}^{1}$ & ${ }_{1}^{1}$ & $i$ & $i$ & - & : & $i$ & $i$ & $i$ & 1 & $i$ & : & $i$ & $i$ & : & $\therefore$ & 1 \\
\hline 1.3 & Goal setting (outcomes) & & 1 & - & & 1 & & 1 & $i$ & 1 & 1 & 1 & - & 1 & : & 1 & 1 & $i$ & $i$ & : \\
\hline $\begin{array}{l}1.4 \\
1.5\end{array}$ & $\begin{array}{c}\text { Action planning } \\
\text { Review behaviour goal(s) }\end{array}$ & 1 & i & & & $i$ & 1 & : & : & 1 & $\begin{array}{l}1 \\
1\end{array}$ & : & 1 & : & : & $=$ & : & : & : & - \\
\hline 1.6 & $\begin{array}{l}\text { Discrepancy between current } \\
\text { behaviour and goal }\end{array}$ & & 1 & 1 & & - & 1 & & & - & 1 & - & - & - & - & - & - & - & - & - \\
\hline 1.7 & $\begin{array}{l}\text { Review outcome goal(s) } \\
\text { Rever }\end{array}$ & & 1 & & & & & & - & - & - & - & - & - & - & 1 & - & - & - & - \\
\hline $\begin{array}{l}1.8 \\
2.2\end{array}$ & $\begin{array}{l}\text { Feedback on behtentiour } \\
\text { Ceniour }\end{array}$ & $i$ & $i$ & $i$ & $i$ & $i$ & $=$ & $i$ & - & $i$ & $i$ & $i$ & $=$ & $i$ & 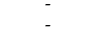 & $=$ & $=$ & $=$ & 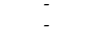 & $=$ \\
\hline 2.3 & Self-monitoring of behaviour & & & 1 & 1 & 1 & - & - & 1 & 1 & 1 & 1 & 1 & 1 & - & 1 & 1 & - & 1 & - \\
\hline 2.7 & $\begin{array}{l}\text { Feedback on outcome(s) of } \\
\text { behaviour }\end{array}$ & - & 1 & & & & - & - & - & - & - & - & - & 1 & - & 1 & - & - & - & - \\
\hline $\begin{array}{l}3.1 \\
3.2\end{array}$ & $\begin{array}{l}\text { Social suenport (unspecified) } \\
\text { Social support (practical) }\end{array}$ & 1 & 1 & 1 & 1 & 1 & 1 & 1 & 1 & 1 & 1 & 1 & 1 & 1 & 1 & $\begin{array}{l}1 \\
1\end{array}$ & 1 & $\begin{array}{l}1 \\
1\end{array}$ & 1 & 1 \\
\hline 4.1 & $\begin{array}{l}\text { Instruction on how to perform } \\
\text { the behaviour }\end{array}$ & 1 & 1 & 1 & 1 & 1 & 1 & 1 & 1 & 1 & 1 & 1 & 1 & 1 & 1 & 1 & 1 & 1 & - & 1 \\
\hline 5.1 & $\begin{array}{c}\text { Information on } \\
\text { health consequences }\end{array}$ & - & - & - & - & - & 1 & - & - & 1 & 1 & 1 & 1 & - & 1 & - & & & & 1 \\
\hline 5.4 & $\begin{array}{l}\text { Monitoring of } \\
\text { emotional consequences }\end{array}$ & & & & & & & & & 1 & & - & - & - & - & - & - & - & - & - \\
\hline 5.6 & $\begin{array}{l}\text { emotional consequences } \\
\text { Information on } \\
\text { emotional consequences }\end{array}$ & - & & - & - & - & - & - & - & - & . & . & 1 & - & - & - & - & - & - & - \\
\hline 6.1 & $\begin{array}{l}\text { emotional consequences } \\
\text { Demonstration of the behaviour }\end{array}$ & - & & - & - & - & 1 & - & - & - & . & - & 1 & - & 1 & - & 1 & - & - & 1 \\
\hline 6.2 & $\begin{array}{l}\text { Social comparison } \\
\text { Prompts }\end{array}$ & $i$ & : & - & : & - & 1 & - & - & i & - & - & - & - & - & - & - & - & - & - \\
\hline 7.3 & Reduce prompts/cues & & - & - & & 1 & $=$ & $=$ & - & - & - & - & & - & - & & - & - & - & - \\
\hline 8.1 & Behavioural practice/ /rehearsal & - & - & - & - & - & - & - & - & - & i & i & 1 & - & 1 & 1 & - & - & - & - \\
\hline $\begin{array}{l}8.7 \\
9.1\end{array}$ & $\begin{array}{l}\text { Graded task } \\
\text { Credible source }\end{array}$ & $i$ & $i$ & $i$ & $i$ & $i$ & $i$ & $i$ & $i$ & $i$ & $\begin{array}{l}1 \\
1\end{array}$ & $\begin{array}{l}1 \\
1\end{array}$ & $\begin{array}{l}1 \\
1\end{array}$ & $i$ & $i$ & $i$ & $i$ & $i$ & $i$ & $i$ \\
\hline 9.2 & $\begin{array}{l}\text { Pros and cons } \\
\text { Solffinconitiog }\end{array}$ & & 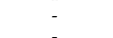 & 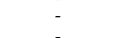 & & 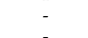 & 1 & 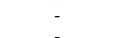 & 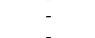 & - & 1 & 1 & 1 & - & - & - & - & - & - & - \\
\hline 12.3 & Avoidance/reducing exposure & - & - & - & . & 1 & - & - & - & - & - & 1 & 1 & - & - & - & - & - & - & - \\
\hline \multirow[t]{2}{*}{13.2} & $\begin{array}{l}\text { to cues sor the behaviour } \\
\text { Framing/reframing }\end{array}$ & - & - & - & - & - & - & - & - & - & - & - & 1 & - & - & - & - & - & - & - \\
\hline & Total number & 7 & 10 & 8 & 7 & 10 & 10 & 5 & 5 & 13 & 13 & 13 & 17 & 8 & 6 & 10 & 6 & 5 & 4 & 6 \\
\hline
\end{tabular}




\subsection{Dietary Behaviour Outcomes}

Most studies assessed behaviour change by measuring differences in the dietary intake at the baseline and follow up. The most frequently reported dietary outcomes were changes in portions of fruit and vegetables, fibre and energy intake. Only seven studies provided the data suitable for meta-analysis [33-35,37,42,43,54]. Certainty of evidence is detailed in Supplementary Tables S2 and S3, and the analyses are presented in Supplementary Figures S1-S8.

For the dietary intake, we found no difference in energy, fruit, vegetable, fruit and vegetables, and fibre intake between the intervention group and control at 6 and 12 months. For adherence, three studies reported a Diet Quality Index. The analysis showed that dietary interventions compared to the control is likely to improve the Diet Quality Index (mean difference 3.62, 95\% CI 1.95 to 5.30; three studies; 719 participants; moderate-certainty evidence) at 12 months. See Supplementary Figure S6-Analysis 1.6. Two studies were excluded from the analysis. One study reported the quality of the diet using a different index [40] and one study used a different scoring system [49].

For anthropometric measures, we found no difference in the body weight and waist circumference, but at 12 months, the dietary intervention versus control probably led to a slight decrease in the body mass index (mean difference $-0.79 \mathrm{~kg} / \mathrm{m} 2,95 \% \mathrm{CI}-1.50$ to -0.07 ; four studies; 777 participants; moderate-certainty evidence). Supplementary Figure S8-Analysis 2.2.

\subsection{Mediators of Behaviour}

Although several constructs were identified and used in the interventions, most included studies did not measure the mediators related to the theory. Therefore, it was not possible to determine if changes in outcomes were due to changes in the mediator variables. The included studies were designed to understand how an intervention changes behaviour, but not what aspect of the theory is positively related to the behaviour change and reported outcomes (i.e., the RCTs tended to be pragmatic rather than explanatory trials).

Only four studies attempted to address the mediators of behaviour [33-35,49]. In paperbased interventions with automated messages, self-efficacy for eating more fruit and vegetables was assessed at the baseline [33]. The study showed that more than $60 \%$ of the participants in both groups were already in very sure to an extremely sure stage of eating more fruit and vegetables at the start of the intervention.

One study using a tailored workbook with targeted telephone counselling reported self-efficacy for healthy eating [34]. This study showed that at 12 months, the number of people in the intervention group who were not sure at all about healthy eating decreased from $4.5 \%$ to $2.6 \%$, those who were a little sure decreased from $2.3 \%$ to $1.3 \%$, those who were somewhat sure decreased from $24.7 \%$ to $22.1 \%$, those who were very sure increased from $43.8 \%$ to $57.1 \%$, and those extremely sure decreased from $24.7 \%$ to $16.9 \%$. Similar numbers were reported for the attrition control group who received a general workbook and general health recommendations through telephone counselling. One study [35] reported self-efficacy for adhering to a healthy weight loss diet as a score (very unsure $=5$ to very sure $=1$ ). At 12 months compared to the baseline, the score for the intervention group changed from 1.9 (standard deviation (SD) 0.8) to 2.1 (SD 0.9), while in the control group the score changed from 2.1 (SD 0.9) to 2.3 (SD 0.8). To provide formal tests of process evaluation, such results need analysing using inferential statistics.

The readiness to change defined based on the TTM as a precontemplation, contemplation, action, and maintenance stage was assessed in three studies. One study on a paper-based intervention with automated messages assessed the readiness to improve fruit and vegetable intake at the baseline and showed that around $60 \%$ of the participants were already in the preparation stage for change and around 30\% in the action stage in both groups [33]. Two paper-based interventions with telephone support assessed the readiness to change the diet. One study [49] reported a difference between the baseline and three months and showed that in the intervention group, the number of people in the precontemplation stage and contemplation change decreased from $28 \%$ to 0 , and from $50 \%$ to $11 \%$ in the preparation stage, while the number of people increased from $22 \%$ to $89 \%$ in 
the action or maintenance stage. In the control group, there was a decrease in the number of people in the pre-contemplation and contemplation stage from $28 \%$ to $22 \%$, increase in the number of people in the preparation stage from $50 \%$ to $61 \%$, and decrease in the number of people in the action or maintenance stage from $22 \%$ to $17 \%$.

In the second study [34] at 12 months, the number of people in the intervention group in the pre-contemplative stage increased from $10.1 \%$ to $19.5 \%$, in the contemplative stage decreased from $84.3 \%$ to $72.7 \%$, and in the preparation stage the number increased from $5.6 \%$ to $7.8 \%$. In the control group, there was an increase in people being in the pre-contemplative stage from $9.7 \%$ to $16.9 \%, 79 \%$ remained in the contemplative stage, and the number of people in the preparation stage decreased from $10.7 \%$ to $3.6 \%$.

The paper-based interventions with tailored automated messages also evaluated the number of practiced goals at 12 months. The study reported that in the intervention group, the number of people with no practicing goal decreased from 116 to 59 , and the number of people practicing two goals increased from 0 to 70 , and the number of three goals from 0 to 15. The control group who received only general materials showed that the number of people with no practicing goal decreased from 115 to 90 , the number of people practicing two goals increased from 0 to 41, and the number of people practising three goals from 0 to 7 [33].

\section{Discussion}

This systematic review established the use and implementation of behaviour change theories in dietary interventions for cancer survivors. SCT and TTM were the most frequently used theories, similar to other reviews of health behaviour change [12,22]. While all the included studies used theory to inform their intervention, due to the failure of most studies to measure constructs from such theories, it was impossible to determine how or if changes in targeted constructs, such as self-efficacy or readiness to change, mediated the effects of interventions on the reported outcomes. Therefore, only the meta-analyses of behavioural outcomes were conducted.

These analyses were based on telephone, group, and mailed interventions that used SCT, TTM or a combination of both. All the interventions also included the following $\mathrm{BCTs}$ - instructions on how to perform the behaviour, credibility of the source, and social support. Moreover, these meta-analyses are mostly applicable to the female population after breast cancer due to the prevalence of breast cancer studies.

The meta-analyses showed little or no difference in dietary outcomes, body weight, and waist circumference, as well as small changes in body mass index and likely an improvement in the diet quality index. A previous systematic review of dietary and physical activity interventions for cancer survivors applying SCT to the interventions concluded that SCT-based interventions provide promising results. However, no metaanalysis was conducted [27]. Weak changes in the dietary behaviour in studies using SCT and TTM in this review are not dissimilar from findings in the Cochrane review, which included also studies that did not incorporate any behaviour change theory [30].

Uncertain evidence and inconsistent findings may be related to many limitations in the interventions that remain unaddressed. Criticism of the conduct of behavioural interventions and suggestions for the best practice were reported some time ago [24-26,55]. However, many interventions aimed at people after cancer still do not adhere to these standards.

\subsection{Intervention Design}

It has been demonstrated that interventions addressing ethical, cultural, and environmental requirements in the targeted population are more effective [14]. In our review, one study considered the ethical differences in the study population and appropriately tailored resources [33] and one study developed an evidence-based programme targeting specifically Hispanic breast cancer survivors [37]. To design the tailored intervention for a specific population requires public involvement, piloting, and feasibility testing, which are often lacking in interventions. The possible reasons for the limited developmental work are funding and time constraints of the research. However, it has been recognised that 
piloting and feasibility testing is an important step to enhance the development of complex interventions, and Medical Research Council (MRC) guidance exists on the development and evaluation of complex interventions in healthcare [56]. In this review, only few interventions on breast cancer $[33,37,42]$ and endometrial cancer [40] have been based on extensive developmental work before conducting the intervention.

\subsection{Description of the Intervention}

Currently, there is no standard way to report how theory is incorporated into dietary interventions and what behavioural aspects are addressed. Interventions claim to use theory, but fail to describe that theory in sufficient detail, and thus do not add to the knowledge accumulation and informed development of future studies. In essence, studies are pragmatic attempts to change behaviour, rather than explanatory trials attempting to understand the underlying theoretical mechanisms. For instance, it has been previously shown, that only $44 \%$ of 34 RCTs stated a theoretical basis for the intervention development [57] and only $50 \%$ of protocol-specified behaviour change techniques were reported in studies from Cochrane reviews on smoking cessation [58]. In our review, $58 \%$ of the studies did not publish a protocol, and $37 \%$ poorly reported on the intervention design, behaviour change theory implementation, and BCTs.

Similar to our review, others have also attempted to map BCTs used in intervention studies based on descriptions provided by the study authors, so as to report on the BCT use as accurately as possible [22,59]. An extended version of the CONSORT statement for reporting randomised trials of social and psychological interventions [60] exists. However, it omits to include details on how to report on the behaviour change theory implemented in the interventions and to address components based on BCT taxonomy. Hence, having consistent guidance for interventions on how and what to report about the theory incorporated in an intervention in the protocol and publication can bring clarity as well as improve the transparency of interventions [24].

\subsection{Outcomes Reporting}

The standardisation of outcomes reporting would allow for more robust pooling of data in the meta-analyses. In this review, studies reported data at different time points and presented data in different ways. Therefore, only seven studies out of 19 were included in the meta-analyses. If more studies would report at 3,6, and 12 months intervals and provided more detailed descriptive data, it would improve the opportunity to conduct data synthesis and an effective comparison. For instance, exercise interventions for cancer survivors are conducted and reported reasonably consistently, and thus the meta-analyses with large numbers of studies have been conducted [22]. Researchers have been able to demonstrate that interventions were effective in achieving a modest increase in physical activity at 3 months. Furthermore, developing a core set of outcomes as "an agreed, standardised and minimum set of outcomes that should be measured and reported in all clinical trials for a specific health condition" could improve the conduct of the meta-analyses [61] and thus provide fuller insight into the efficacy and effectiveness of interventions.

\subsection{Assessment of the Behaviour Change Theory in Interventions}

Although most interventions used theory, the majority of interventions did not assess the mediators of behaviour change related to theory (e.g., readiness to change) nor theory constructs (e.g., self-efficacy). Our review highlights that research teams are primarily focused on outcome evaluation and do not routinely conduct (or at least report) process evaluations to check that their interventions are working as per the design.

Only four studies out of 19 reported measures of such mediators. However, inconsistency in measurement meant it was not possible to meta-analyse these studies. The readiness to change and self-efficacy are important mediators that should be reported at the baseline. Grimmett et al. (2019) highlighted that if people enrolled in interventions are highly motivated to change their behaviour, a type two error occurs in the outcomes 
measurement irrespective of the group allocation, and small differences between the groups observed can mask the true effect of the intervention [22]. This has been demonstrated in the included studies which reported on self-efficacy and readiness to change at the baseline. These studies indicated that most participants are in the preparation or action stage or are fairly confident to make a change at the start of the intervention, and where groups are compared over time, small differences are observed.

Rejeski et al. (2000) also suggested that studies should report a readiness to change over time to provide the indication of success of the intervention [62]. Participants' attitudes are likely to change during the intervention based on how they perceive the intervention to be difficult, and how they cope with it, and their level of motivation. This indicates that unless the mediators of behaviour such as self-efficacy and readiness to change are reported over time, the study's outcomes can be misleading.

In summary, it remains unclear what theory works best or what constructs meaningfully influence the participants' behaviour in dietary interventions for cancer survivors. Limitations such as lack of details on the applied theory, BCTs, and process evaluation in studies presented in this review prevent us from drawing any conclusion on the effectiveness of incorporating specific theories in interventions. Hence, better consistency in the design, evaluation, and reporting of dietary interventions for cancer survivors is required, in order to demonstrate not only how the implementation of theory supports the interventions, but also determine what is a cumulative effect of interventions on the targeted outcomes.

\subsection{Limitations}

Due to the limited consistency in reporting, all 19 authors would have had to be contacted to provide the details lacking on the behaviour change theory and BCT techniques used. We were unable to contact all the authors and hence, the review is based on publicly available information from studies and study protocols and thus highlights gaps in the quality of study reporting.

\section{Conclusions}

Currently, uncertainty exists on the impact of behaviour change theories implemented in dietary interventions for cancer survivors. There is a need for interdisciplinary work in the design, assessment, and monitoring of interventions in order to allow for the appropriate selection of theories, incorporation of the theory in the intervention design, as well as adequate reporting and testing of the theory to build up valid and consistent evidence. Active collaboration with a psychologist during the planning process and conduct of the trials may help in mitigating a number of issues discussed in this review and improve the efficacy of interventions.

Supplementary Materials: The following are available online at https:/ / www.mdpi.com/2072-664 3/13/2/612/s1. Table S1: Search Strategy; Table S2: GRADE PRO table for dietary outcomes; Table S3: GRADE PRO table for anthropometry outcomes; Figure S1: Mean energy intake; Figure S2: Mean fruit intake; Figure S3: Mean veg etable intake; Figure S4: Mean fruit and vegetable intake; Figure S5: Mean fibre intake; Figure S6: Diet Quality ndex; Figure S7: Mean weight; Figure S8: Mean Body Mass Index.

Author Contributions: Conceptualisation has been completed by J.S. and S.B., J.S., S.B., A.M.S., C.T., and R.C. developed the protocol for the review and agreed on the methodology; J.S. and S.B. conducted the searches; J.S. and A.M.S. completed the selection of the studies; data extraction was completed by J.S. and $20 \%$ was double checked by each co-author; J.S. and S.B. conducted the formal analysis of the data; J.S. drafted the original manuscript; S.B., A.M.S., Richard Cooke, and C.T. significantly contributed to amending and editing of the final version. All authors have read and agreed to the published version of the manuscript. 
Funding: This research was funded by the Research Impact Scholarship Award provided by the Division of Development and Alumni Relations of the University of Manchester, and by the National Institute for Health Research (NIHR) as part of Professor Todd's Senior Investigator award. The views expressed are those of the authors and not necessarily those of the NIHR or the Department of Health and Social Care or its partners.

Institutional Review Board Statement: Not applicable.

Informed Consent Statement: Not applicable.

Conflicts of Interest: The authors declare no conflict of interest. The funders had no role in the design of the study; in the collection, analyses, or interpretation of data; in the writing of the manuscript, or in the decision to publish the results.

\section{References}

1. National Cancer Research Institute. Definitions. 2021. Available online: https://cancercontrol.cancer.gov/ocs/statistics\# definition-survivorship (accessed on 16 January 2021).

2. Vergnaud, A.C.; Romaguera, D.; Peeters, P.H.; Van Gils, C.H.; Chan, D.S.M.; Romieu, I.; Freisling, H.; Ferrari, P.; Clavel-Chapelon, F.; Fagherazzi, G.; et al. Adherence to the World Cancer Research Fund/American Institute for Cancer Research guidelines and risk of death in Europe: Results from the European Prospective Investigation into Nutrition and Cancer cohort study. Am. J. Clin. Nutr. 2013, 97, 1107-1120. [CrossRef]

3. Schwedhelm, C.; Boeing, H.; Hoffmann, G.; Aleksandrova, K.; Schwingshackl, L. Effect of diet on mortality and cancer recurrence among cancer survivors: A systematic review and meta-analysis of cohort studies. Nutr. Rev. 2016, 74, 737-748. [CrossRef]

4. Demark-Wahnefried, W.; Aziz, N.M.; Rowland, J.H.; Pinto, B.M. Riding the Crest of the Teachable Moment: Promoting Long-Term Health After the Diagnosis of Cancer. J. Clin. Oncol. 2005, 23, 5814-5830. [CrossRef]

5. Bluethmann, S.M.; Basen-Engquist, K.; Vernon, S.W.; Cox, M.G.; Gabriel, K.P.; Stansberry, S.A.; Carmack, C.L.; Blalock, J.A.; Demark-Wahnefried, W. Grasping the 'teachable moment': Time since diagnosis, symptom burden and health behaviors in breast, colorectal and prostate cancer survivors. Psycho-Oncology 2015, 24, 1250-1257. [CrossRef] [PubMed]

6. $\quad$ Pinto, B.M.; Maruyama, N.C.; Clark, M.M.; Cruess, D.G.; Park, E.; Roberts, M. Motivation to Modify Lifestyle Risk Behaviors in Women Treated for Breast Cancer. Mayo Clin. Proc. 2002, 77, 122-129. [CrossRef]

7. Smith, L.; Croker, H.; Fisher, A.; Williams, K.; Wardle, J.; Beeken, R.J. Cancer survivors' attitudes towards and knowledge of physical activity, sources of information, and barriers and facilitators of engagement: A qualitative study. Eur. J. Cancer Care 2017, 26, e12641. [CrossRef]

8. Sremanakova, J.; Jones, D.; Cooke, R.; Burden, S. Exploring Views of Healthcare Professionals, Researchers, and People Living with and beyond Colorectal Cancer on a Healthy-Eating and Active Lifestyle Resource. Nutrients 2019, 11, 2482. [CrossRef] [PubMed]

9. Wright, S.J.; Gibson, D.; Eden, M.; Lal, S.; Todd, C.; Ness, A.; Burden, S. What are colorectal cancer survivors' preferences for dietary advice? A best-worst discrete choice experiment. J. Cancer Surviv. 2017, 11, 782-790. [CrossRef]

10. Avery, L.; Flynn, D.; Van Wersch, A.; Sniehotta, F.F.; Trenell, M.I. Changing Physical Activity Behavior in Type 2 Diabetes: A systematic review and meta-analysis of behavioral interventions. Diabetes Care 2012, 35, 2681-2689. [CrossRef]

11. Bartlett, Y.K.; Sheeran, P.; Hawley, M.S. Effective behaviour change techniques in smoking cessation interventions for people with chronic obstructive pulmonary disease: A meta-analysis. Br. J. Health Psychol. 2013, 19, 181-203. [CrossRef]

12. Lara, J.; Evans, E.H.; O’Brien, N.; Moynihan, P.J.; Meyer, T.D.; Adamson, A.J.; Errington, L.; Sniehotta, F.F.; White, M.; Mathers, J.C. Association of behaviour change techniques with effectiveness of dietary interventions among adults of retirement age: A systematic review and meta-analysis of randomised controlled trials. BMC Med. 2014, 12, 177. [CrossRef]

13. McEachan, R.R.C.; Conner, M.; Taylor, N.J.; Lawton, R.J. Prospective prediction of health-related behaviours with the Theory of Planned Behaviour: A meta-analysis. Health Psychol. Rev. 2011, 5, 97-144. [CrossRef]

14. Will, J.C.; Farris, R.P.; Sanders, C.G.; Stockmyer, C.K.; Finkelstein, E.A. Health Promotion Interventions for Disadvantaged Women: Overview of the WISEWOMAN Projects. J. Womens Health 2004, 13, 484-502. [CrossRef] [PubMed]

15. Glanz, K.; Bishop, D.B. The Role of Behavioral Science Theory in Development and Implementation of Public Health Interventions. Annu. Rev. Public Health 2010, 31, 399-418. [CrossRef] [PubMed]

16. Noar, S.M.; Benac, C.N.; Harris, M.S. Does tailoring matter? Meta-analytic review of tailored print health behavior change interventions. Psychol. Bull. 2007, 133, 673-693. [CrossRef]

17. Sheeran, P.; Klein, W.M.; Rothman, A.J. Health Behavior Change: Moving from Observation to Intervention. Annu. Rev. Psychol. 2017, 68, 573-600. [CrossRef]

18. Bandura, A. Health promotion from the perspective of social cognitive theory. Psychol. Health 1998, 13, 623-649. [CrossRef]

19. Prochaska, J.O.; DiClemente, C.C.; Norcross, J.C. In search of how people change: Applications to addictive behaviors. Am. Psychol. 1992, 47, 1102-1114. [CrossRef]

20. Rosenstock, I.M. Why people use health services. Milbank Mem. Fund Q. 1966, 44, 94-127. [CrossRef] 
21. Glanz, K. Theory at a Glance: A Guide for Health Promotion Practice; US Department of Health and Human Services, Public Health Service, National Institute of Health: USA, 1997; Volume 97.

22. Grimmett, C.; Corbett, T.; Brunet, J.; Shepherd, J.; Pinto, B.M.; May, C.R.; Foster, C. Systematic review and meta-analysis of maintenance of physical activity behaviour change in cancer survivors. Int. J. Behav. Nutr. Phys. Act. 2019, 16, 37. [CrossRef]

23. Michie, S.; Carey, R.N.; Johnston, M.; Rothman, A.J.; De Bruin, M.; Kelly, M.P.; Connell, L.E. From Theory-Inspired to Theory-Based Interventions: A Protocol for Developing and Testing a Methodology for Linking Behaviour Change Techniques to Theoretical Mechanisms of Action. Ann. Behav. Med. 2018, 52, 501-512. [CrossRef] [PubMed]

24. Michie, S.; Johnston, M. Theories and Techniques of Behaviour Change: Developing a Cumulative Science of Behaviour Change. Health Psychol. Rev. 2012, 6, 1-6. [CrossRef]

25. Michie, S.; West, R.; Sheals, K.; A Godinho, C. Evaluating the effectiveness of behavior change techniques in health-related behavior: A scoping review of methods used. Transl. Behav. Med. 2018, 8, 212-224. [CrossRef]

26. Noar, S.M.; Zimmerman, R.S. Health Behavior Theory and cumulative knowledge regarding health behaviors: Are we moving in the right direction? Health Educ. Res. 2005, 20, 275-290. [CrossRef]

27. Stacey, F.G.; James, E.L.; Chapman, K.; Courneya, K.S.; Lubans, D.R. A systematic review and meta-analysis of social cognitive theory-based physical activity and/or nutrition behavior change interventions for cancer survivors. J. Cancer Surviv. 2015, 9, 305-338. [CrossRef]

28. Michie, S.; Richardson, M.; Johnston, M.; Abraham, C.; Francis, J.; Hardeman, W.; Eccles, M.P.; Cane, J.; Wood, C.E. The Behavior Change Technique Taxonomy (v1) of 93 Hierarchically Clustered Techniques: Building an International Consensus for the Reporting of Behavior Change Interventions. Ann. Behav. Med. 2013, 46, 81-95. [CrossRef] [PubMed]

29. Graves, K.D. Social cognitive theory and cancer patients' quality of life: A meta-analysis of psychosocial intervention components. Health Psychol. 2003, 22, 210-219. [CrossRef]

30. Burden, S.; Jones, D.J.; Sremanakova, J.; Sowerbutts, A.M.; Lal, S.; Pilling, M.; Todd, C. Dietary interventions for adult cancer survivors. Cochrane Database Syst. Rev. 2019. [CrossRef] [PubMed]

31. Higgins, J.; Altman, D.; Sterne, J.; Higgins, J.P.T.; Green, S. (Eds.) Cochrane Handbook for Systematic Reviews of Interventions Version 5.1; (updated March 2011); Cochrane: London, UK, 2011; Available online: training.cochrane.org/handbook (accessed on 16 January 2021).

32. Moher, D.; Liberati, A.; Tetzlaff, J.; Altman, D.G.; The PRISMA Group. Preferred Reporting Items for Systematic Reviews and Meta-Analyses: The PRISMA Statement. PLoS Med. 2009, 6, e1000097. [CrossRef]

33. Demark-Wahnefried, W.; Clipp, E.C.; Lipkus, I.M.; Lobach, D.; Snyder, D.C.; Sloane, R.; Peterson, B.; Macri, J.M.; Rock, C.L.; McBride, C.M.; et al. Main Outcomes of the FRESH START Trial: A Sequentially Tailored, Diet and Exercise Mailed Print Intervention Among Breast and Prostate Cancer Survivors. J. Clin. Oncol. 2007, 25, 2709-2718. [CrossRef]

34. Demark-Wahnefried, W.; Clipp, E.C.; Morey, M.C.; Pieper, C.F.; Sloane, R.; Snyder, D.C.; Cohen, H.J. Lifestyle Intervention Development Study to Improve Physical Function in Older Adults With Cancer: Outcomes From Project LEAD. J. Clin. Oncol. 2006, 24, 3465-3473. [CrossRef]

35. Demark-Wahnefried, W.; Jones, L.W.; Snyder, D.C.; Sloane, R.J.; Kimmick, G.G.; Hughes, D.C.; Badr, H.J.; Miller, P.E.; Burke, L.E.; Lipkus, I.M. Daughters and Mothers Against Breast Cancer (DAMES): Main outcomes of a randomized controlled trial of weight loss in overweight mothers with breast cancer and their overweight daughters. Cancer 2014, 120, 2522-2534. [CrossRef] [PubMed]

36. Djuric, Z.; DiLaura, N.M.; Jenkins, I.; Darga, L.; Jen, C.K.-L.; Mood, D.; Bradley, E.; Hryniuk, W.M. Combining Weight-Loss Counseling with the Weight Watchers Plan for Obese Breast Cancer Survivors. Obes. Res. 2002, 10, 657-665. [CrossRef] [PubMed]

37. Greenlee, H.; Gaffney, A.O.; Aycinena, A.C.; Koch, P.; Contento, I.; Karmally, W.; Richardson, J.M.; Lim, E.; Tsai, W.-Y.; Crew, K.; et al. ¡Cocinar Para Su Salud!: Randomized Controlled Trial of a Culturally Based Dietary Intervention among Hispanic Breast Cancer Survivors. J. Acad. Nutr. Diet. 2015, 115, 709-723.e3. [CrossRef] [PubMed]

38. Harrigan, M.; Cartmel, B.; Loftfield, E.; Sanft, T.; Chagpar, A.B.; Zhou, Y.; Playdon, M.; Li, F.; Irwin, M.L. Randomized Trial Comparing Telephone Versus In-Person Weight Loss Counseling on Body Composition and Circulating Biomarkers in Women Treated for Breast Cancer: The Lifestyle, Exercise, and Nutrition (LEAN) Study. J. Clin. Oncol. 2016, 34, 669-676. [CrossRef] [PubMed]

39. Hawkes, A.L.; Chambers, S.K.; Pakenham, K.I.; Patrao, T.A.; Baade, P.D.; Lynch, B.M.; Aitken, J.F.; Meng, X.; Courneya, K.S. Effects of a Telephone-Delivered Multiple Health Behavior Change Intervention (CanChange) on Health and Behavioral Outcomes in Survivors of Colorectal Cancer: A Randomized Controlled Trial. J. Clin. Oncol. 2013, 31, 2313-2321. [CrossRef]

40. Koutoukidis, D.A.; Beeken, R.J.; Manchanda, R.; Burnell, M.; Ziauddeen, N.; Michalopoulou, M.; Knobf, M.T.; Lanceley, A. Diet, physical activity, and health-related outcomes of endometrial cancer survivors in a behavioral lifestyle program: The Diet and Exercise in Uterine Cancer Survivors (DEUS) parallel randomized controlled pilot trial. Int. J. Gynecol. Cancer 2019, 29, 531-540. [CrossRef]

41. Morey, M.C.; Snyder, D.C.; Sloane, R.; Cohen, H.J.; Peterson, B.; Hartman, T.J.; Miller, P.; Mitchell, D.C.; Demark-Wahnefried, W. Effects of Home-Based Diet and Exercise on Functional Outcomes Among Older, Overweight Long-term Cancer Survivors. JAMA 2009, 301, 1883-1891. [CrossRef]

42. Pierce, J.P.; Natarajan, L.; Caan, B.J.; Parker, B.A.; Greenberg, E.R.; Flatt, S.W.; Rock, C.L.; Kealey, S.; Al-Delaimy, W.K.; Bardwell, W.A.; et al. Influence of a Diet Very High in Vegetables, Fruit, and Fiber and Low in Fat on Prognosis Following Treatment for Breast Cancer. JAMA 2007, 298, 289-298. [CrossRef] 
43. Von Gruenigen, V.; Frasure, H.; Kavanagh, M.B.; Janata, J.; Waggoner, S.; Rose, P.; Lerner, E.; Courneya, K.S. Survivors of uterine cancer empowered by exercise and healthy diet (SUCCEED): A randomized controlled trial. Gynecol. Oncol. 2012, 125, 699-704. [CrossRef] [PubMed]

44. Higgins, J.P.T.; Altman, D.G.; Gøtzsche, P.C.; Jüni, P.; Moher, D.; Oxman, A.D.; Savović, J.; Schulz, K.F.; Weeks, L.; Sterne, J.A.C.; et al. The Cochrane Collaboration's tool for assessing risk of bias in randomised trials. BMJ 2011, 343, d5928. [CrossRef] [PubMed]

45. Guyatt, G.; Oxman, A.D.; Akl, E.A.; Kunz, R.; Vist, G.; Brozek, J.; Norris, S.; Falck-Ytter, Y.; Glasziou, P.; Debeer, H. GRADE guidelines: 1. Introduction-GRADE evidence profiles and summary of findings tables. J. Clin. Epidemiol. 2011, 64, 383-394. [CrossRef]

46. RevMan R, version 5.4.1; The Nordic Cochrane Centre, The Cochrane Collaboration: Oxford, UK, 2014.

47. Van Blarigan, E.L.; Kenfield, S.A.; Chan, J.M.; Van Loon, K.; Paciorek, A.; Zhang, L.; Chan, H.; Savoie, M.B.; Bocobo, A.G.; Liu, V.N.; et al. Feasibility and acceptability of a web-based dietary intervention with text messages for colorectal cancer: A randomized pilot trial. Cancer Epidemiol. Biomark. Prev. 2020, 29, 752-760. [CrossRef]

48. Kanera, I.M.; Willems, R.A.; Bolman, C.A.W.; Mesters, I.; Verboon, P.; Lechner, L. Long-term effects of a web-based cancer aftercare intervention on moderate physical activity and vegetable consumption among early cancer survivors: A randomized controlled trial. Int. J. Behav. Nutr. Phys. Act. 2017, 14, 1-13. [CrossRef]

49. Kim, S.H.; Shin, M.S.; Lee, H.S.; Lee, E.S.; Ro, J.S.; Kang, H.S.; Kim, S.W.; Lee, W.H.; Kim, H.S.; Kim, C.J.; et al. Randomized Pilot Test of a Simultaneous Stage-Matched Exercise and Diet Intervention for Breast Cancer Survivors. Oncol. Nurs. Forum 2011, 38, E97-E106. [CrossRef]

50. Parekh, N.; Jiang, J.; Buchan, M.; Meyers, M.; Gibbs, H.; Krebs, P. Nutrition Literacy among Cancer Survivors: Feasibility Results from the Healthy Eating and Living Against Breast Cancer (HEAL-BCa) Study: A Pilot Randomized Controlled Trial. J. Cancer Educ. 2018, 33, 1239-1249. [CrossRef]

51. Sheppard, V.B.; Hicks, J.; Makambi, K.H.; Hurtado-De-Mendoza, A.; Demark-Wahnefried, W.; Adamscampbell, L.L. The feasibility and acceptability of a diet and exercise trial in overweight and obese black breast cancer survivors: The Stepping STONE study. Contemp. Clin. Trials 2016, 46, 106-113. [CrossRef] [PubMed]

52. Yun, Y.H.; Kim, Y.A.; Lee, M.K.; Sim, J.A.; Nam, B.-H.; Kim, S.; Lee, E.S.; Noh, D.-Y.; Lim, J.-Y.; Kim, S.; et al. A randomized controlled trial of physical activity, dietary habit, and distress management with the Leadership and Coaching for Health (LEACH) program for disease-free cancer survivors. BMC Cancer 2017, 17, 298. [CrossRef]

53. Zick, S.M.; Colacino, J.; Cornellier, M.; Khabir, T.; Surnow, K.; Djuric, Z. Fatigue reduction diet in breast cancer survivors: A pilot randomized clinical trial. Breast Cancer Res. Treat. 2017, 161, 299-310. [CrossRef]

54. Zuniga, K.E.; Parma, D.L.; Muñoz, E.; Spaniol, M.; Wargovich, M.; Ramirez, A.G. Dietary intervention among breast cancer survivors increased adherence to a Mediterranean-style, anti-inflammatory dietary pattern: The Rx for Better Breast Health Randomized Controlled Trial. Breast Cancer Res. Treat. 2018, 173, 145-154. [CrossRef] [PubMed]

55. Dombrowski, S.U.; Sniehotta, F.F.; Avenell, A.; Coyne, J.C. Current issues and future directions inPsychology and Health: Towards a cumulative science of behaviour change: Do current conduct and reporting of behavioural interventions fall short of best practice? Psychol. Health 2007, 22, 869-874. [CrossRef]

56. Craig, P.; Dieppe, P.; MacIntyre, S.; Michie, S.; Nazareth, I.; Petticrew, M. Developing and evaluating complex interventions: The new Medical Research Council guidance. BMJ 2008, 337, a1655. [CrossRef] [PubMed]

57. Kok, G.; Schaalma, H.; Ruiter, R.A.C.; Van Empelen, P.; Brug, J. Intervention Mapping: Protocol for Applying Health Psychology Theory to Prevention Programmes. J. Health Psychol. 2004, 9, 85-98. [CrossRef]

58. Lorencatto, F.; West, R.; Stavri, Z.; Michie, S. How Well Is Intervention Content Described in Published Reports of Smoking Cessation Interventions? Nicotine Tob. Res. 2012, 15, 1273-1282. [CrossRef]

59. Balhareth, A.; Aldossary, M.Y.; McNamara, D. Impact of physical activity and diet on colorectal cancer survivors' quality of life: A systematic review. World J. Surg. Oncol. 2019, 17, 153. [CrossRef]

60. Montgomery, P.; Grant, S.; Mayo-Wilson, E.; Macdonald, G.; Michie, S.; Hopewell, S.; Moher, D. Reporting randomised trials of social and psychological interventions: The CONSORT-SPI 2018 Extension. Trials 2018, 19, 407. [CrossRef]

61. Chiarotto, A.; Ostelo, R.W.; Turk, D.C.; Buchbinder, R.; Boers, M. Core outcome sets for research and clinical practice. Braz. J. Phys. Ther. 2017, 21, 77-84. [CrossRef] [PubMed]

62. Rejeski, W.; Brawley, L.R.; McAuley, E.; Rapp, S. An examination of theory and behavior change in randomized clinical trials. Control. Clin. Trials 2000, 21, S164-S170. [CrossRef] 